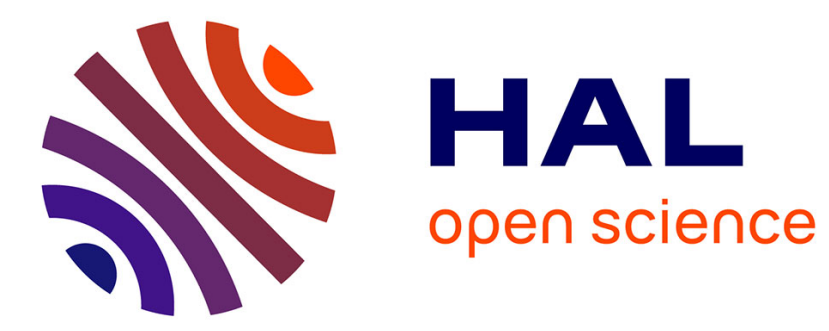

\title{
Afriat's theorem for indivisible goods
}

Francoise Forges, Vincent Iehlé

\section{- To cite this version:}

Francoise Forges, Vincent Iehlé. Afriat's theorem for indivisible goods. 2013. halshs-00870052v1

\section{HAL Id: halshs-00870052}

\section{https://shs.hal.science/halshs-00870052v1}

Preprint submitted on 4 Oct 2013 (v1), last revised 26 Aug 2014 (v2)

HAL is a multi-disciplinary open access archive for the deposit and dissemination of scientific research documents, whether they are published or not. The documents may come from teaching and research institutions in France or abroad, or from public or private research centers.
L'archive ouverte pluridisciplinaire HAL, est destinée au dépôt et à la diffusion de documents scientifiques de niveau recherche, publiés ou non, émanant des établissements d'enseignement et de recherche français ou étrangers, des laboratoires publics ou privés. 


\title{
AFRIAT'S THEOREM FOR INDIVISIBLE GOODS*
}

\author{
Françoise Forges ${ }^{\dagger}$ \\ Vincent Iehlé \\ U. Paris-Dauphine \& IUF \\ U. Paris-Dauphine
}

October 4, 2013

\begin{abstract}
We identify a natural counterpart of the standard GARP for demand data in which goods are all indivisible. We show that the new axiom (DARP, for "discrete axiom of revealed preference") is necessary and sufficient for the rationalization of the data by a well-behaved utility function. Our results complement the main finding of Polisson and Quah, Am. Econ. J.: Micro. 5(1) p.28-34 (2013), who rather minimally modify the original consumer problem with indivisible goods so that the standard GARP still applies.
\end{abstract}

JEL classification numbers: D11,C81.

Keywords: Afriat's theorem, GARP, indivisible goods, rationalization, revealed preference.

\section{INTRODUCTION}

When goods are perfectly divisible, Afriat (1967)'s theorem tells us that the general axiom of revealed preference (GARP) is a necessary and sufficient condition for consumption data to be consistent with utility maximization (see, e.g., Diewert, 1973; Varian, 1982). The proof of the result is fully constructive, namely yields an explicit well-behaved utility function when GARP is satisfied. In the standard formulation of GARP, it is understood that rational preferences are locally nonsatiated. However, in practice, goods are often indivisible and traded in discrete quantities in the field or in the laboratory. In this case, as recently acknowledged by Polisson and Quah (2013) and Fujishige and Yang (2012), local nonsatiation becomes meaningless so that GARP, in its usual form, is no longer a necessary condition of rationalization.

\footnotetext{
${ }^{*}$ We are grateful to Don Brown, Sam Cosaert, Thomas Demuynck and John Quah for helpful comments.

${ }^{\dagger}$ E-mail: Francoise.Forges@dauphine.fr. Address: LEDa \& CEREMADE, Université Paris-Dauphine, Place du Maréchal de Lattre de Tassigny, 75775 Paris Cedex 16, FRANCE.

${ }^{\ddagger}$ E-mail: Vincent.lehle@dauphine.fr. Address: LEDa \& CEREMADE, Université Paris-Dauphine, Place du Maréchal de Lattre de Tassigny, 75775 Paris Cedex 16, FRANCE.
} 
This note proposes an analog of Afriat's theorem when the data consist of finitely many observed prices and consumption bundles that belong to a discrete consumption set. To this aim, we identify a discrete axiom of revealed preference (DARP) and show that it is necessary and sufficient for rationalization of our data. We start by defining a relation of direct preference, exactly as for the standard GARP. More precisely, let $x$ and $x^{\prime}$ be bundles of indivisible goods that have been purchased at price $p$ and $p^{\prime}$ respectively; the bundle $x$ is directly revealed preferred to the bundle $x^{\prime}$ if $x^{\prime}$ was feasible at price $p$ given the consumer's budget. We go on by defining an indirect revealed preference relation as the transitive closure of the previous relation. A set of observations satisfies DARP if, whenever a bundle $x$ is indirectly revealed preferred to a bundle $x^{\prime}$ the bundle $x+\mathbf{1}$, which is obtained by adding one unit of every good to $x$, is not feasible at price $p^{\prime}$. DARP can thus be described as a discrete analog of GARP, in which the interior of a budget set consists of those bundles that remain in the budget set when all their components increase by one unit. As expected, DARP implies GARP but the reverse is not true.

Our main result (proposition 1) states that DARP, as described above, is a necessary and sufficient condition for the rationalization of a finite set of discrete data by a discrete quasiconcave and monotonic utility function. Surprisingly, the proof can still make use of Afriat's methodology. The key difference is that, extended over a continuous consumption space, our utility function would be flat on a small domain. However, its restriction to integer bundles turns out to be well-behaved, in particular monotonic.

So far, the utility function that we have proposed to rationalize the data when DARP is satisfied is monotonic but not strictly monotonic, while the latter property is specially desirable in the context of indivisible goods. We show (in proposition 2) that a strengthening of DARP, which we denote as DARP*, is necessary and sufficient for rationalization by a strictly monotonic utility function. As in the continuous case, a basic tool to establish propositions 1 and 2 is that DARP and DARP* are equivalent to the cyclical consistency of matrices that are associated to the data. This property is formalized in proposition 3 .

In the standard approach to revealed preference, it is implicitly assumed that the consumer exhausts his revenue at every date, namely, that his revenue is $p \cdot x$ when he purchases the bundle $x$ at price $p$. This assumption, which is sensible when goods are perfectly divisible and the consumer has monotonic preferences, is disputable when goods are only available in discrete quantities. In this case, consumption of a bundle $x$ at price $p$ basically indicates that the consumer's revenue, which is not observed by the analyst, is above $p \cdot x$ and below $p \cdot(x+\mathbf{1})$. Propositions 1 and 2 are formulated in terms of standard budget sets but propositions 4 and 5 extend them, respectively for DARP and DARP*, in order to reflect the possible budget sets of 
the consumer.

\subsection{Related literature}

Polisson and Quah (2013) also address the problem of revealed preferences in the context of indivisible goods but adopt a different approach. To solve the problem that is generated by the lack of meaningful local nonsatiation, Polisson and Quah (2013) allow for an implicit additional consumption good, which can be purchased in continuous quantities. Formally their model of rationality comes close to the standard consumer problem with quasilinear utility where the additional good plays the role of money. They show that this is enough to guarantee that the standard GARP be a necessary and sufficient condition for the existence of a strictly increasing utility function on the discrete consumption space that rationalizes price and demand observations. In Fujishige and Yang (2012), an identical conclusion is obtained without any additional good but the problem related to local nonsatiation is evicted right away by assuming cost efficiency.

Models with continuous goods and money are central in Brown and Calsamiglia (2007) and Sákovics (2013). They introduce an axiom that is stronger than GARP, which they call respectively cyclical monotonicity condition and axiom of revealed valuation. They show that such an axiom is relevant for rationalization by a quasilinear utility when the data consist of finitely many observed prices and bundles of continuous goods. A similar conclusion is reached as a by-product in Echenique et al. (2011a, p.1211). In any case, as soon as the presence of (continuous) money is explicitly acknowledged, imposing discrete quantities for the consumption goods becomes innocuous from a revealed preference perspective and an identical test applies for rationalizing the data.

Recently, Cosaert and Demuynck (2013) have considered the rationalization of observations from finite consumption sets. In this general framework, they identify two variants of GARP, called WMARP and SMARP, that reduce to DARP and DARP* in the standard consumer problem with indivisible goods. It follows that a by-product of their conclusions comes close to our main findings. However, with respect to the problem addressed here, the utility functions we construct behave better than the ones obtained by Cosaert and Demuynck (2013) under WMARP and SMARP and satisfy especially (discrete) quasi-concavity. The reason for this is that we can make full use of the "linear"structure of budget sets and the uniform incrementation by the vector 1 to define the interior of the budgets sets and follow Afriat's methodology. This contrasts with the approach of Cosaert and Demuynck (2013) who posit no assumption, except 
finiteness, on the structure of the choice sets and use therefore a different strategy. ${ }^{1}$

We will make a more precise comparison between our results and the ones of Polisson and Quah (2013) and of Cosaert and Demuynck (2013) once we are equipped with precise definitions, in section 2 .

Our paper is of course also related with studies of revealed preferences that do not focus on competitive budget sets. As recalled e.g. in Mas-Colell et al. (1995, chap.3, p.92), under suitable assumptions, the SARP is a necessary and sufficient condition for the existence of a rationalization in abstract environments of choice. This approach is followed by Echenique et al. (2011b) to deal with invisibilities (see also Chambers and Echenique (2009) in the framework of finite lattices). In this paper we rather follow Afriat's constructive approach, which enables us to check whether the possible rationalization is well-behaved (monotone, concave, etc.).

\subsection{Notations and terminology.}

The vector $\mathbf{1}$ is the characteristic vector of $\mathbb{R}^{K}$ whose components are all equal to 1 ; and for any $\ell=1, \ldots, K, \mathbf{e}^{\ell}$ is the vector of $\mathbb{R}^{K}$ whose component $\ell$ is 1 and remaining components are all 0 . The mapping $u: \mathbb{N}^{K} \rightarrow \mathbb{R}$ is monotonic if for every $x, x^{\prime} \in \mathbb{N}^{K}$ such that $x \gg x^{\prime}, u(x)>u\left(x^{\prime}\right) ; u$ is strictly monotonic if for every $x, x^{\prime} \in \mathbb{N}^{K}$ such that $x>x^{\prime}, u(x)>u\left(x^{\prime}\right)$. Given a set $A \subset \mathbb{N}^{K}$, $\mathbb{1}_{A}$ is the indicator function of $A$, that is, $\mathbb{1}_{A}(x)=1$ if $x \in A$ and $\mathbb{1}_{A}(x)=0$ otherwise; and $A^{c}$ is the complement of $A$ in $\mathbb{N}^{K}$. A set $A \subset \mathbb{N}^{K}$ is discrete convex if for every $x_{1}, \ldots, x_{m} \in A$, with $m \in \mathbb{N}$, and every $\lambda_{1}, \ldots, \lambda_{m} \geq 0$ with $\sum_{i=1}^{m} \lambda_{i}=1$ and $\sum_{i} \lambda_{i} x_{i} \in \mathbb{N}^{K}$ it holds that $\sum_{i} \lambda_{i} x_{i} \in A$. A mapping $u: \mathbb{N}^{K} \rightarrow \mathbb{R}$ is discrete quasi-concave if $\left\{z \in \mathbb{N}^{K}: u(z) \geq k\right\}$ is a discrete convex set for any $k \in \mathbb{R}$. Observe that if $u: \mathbb{R}_{+}^{K} \rightarrow \mathbb{R}$ is quasi-concave then its restriction to $\mathbb{N}^{K}$ is discrete quasi-concave.

\section{RATIONALIZATION AND DARP}

Consider an analyst observing at each date $t=1, \ldots, n$ the bundle $x_{t} \in \mathbb{R}_{+}^{K}$ purchased by a single consumer, and positive prices $p_{t} \in \mathbb{R}_{++}^{K}$. For the sake of the presentation we distinguish consumption constraints from budgetary constraints. The consumption set is $\mathbb{N}^{K}$, and the budget set at any date $t$ is: ${ }^{2}$

$$
B_{t}:=\left\{x \in \mathbb{R}_{+}^{K}: p_{t} \cdot x \leq p_{t} \cdot x_{t}\right\}
$$

\footnotetext{
${ }^{1}$ Interestingly, they apply their results to reappraise the number of inconsistent subjects in past experimental studies that use GARP instead of its discrete/finite variants.

${ }^{2}$ The definition of $B_{t}$ implicitly assumes that the consumer exhausts entirely its revenue $r_{t}$ at $x_{t}$, that is, $p_{t} \cdot x_{t}=r_{t}$. This restriction is relaxed in section 5 .
} 
To assess whether the consumer behaves rationally, the analyst can use this basic data to verify whether the observed choices match the solutions of the standard consumer problem defined by the observed prices at each date and the consumption set.

Definition 1 A utility function $u: \mathbb{N}^{K} \rightarrow \mathbb{R}$ is called a rationalization of the observations $\left(x_{t}, p_{t}\right)_{t=1, \ldots, n}$ if, at each date $t, x_{t}$ solves

$$
\max u(x) \text { subject to } x \in B_{t} \cap \mathbb{N}^{K}
$$

To formulate our axiom we proceed as usual by defining first the direct revealed preference relation, denoted by $R$. The bundle $x_{i}$ is said to be directly revealed preferred to $x_{j}, x_{i} R x_{j}$, if $x_{j} \in B_{i}$. The transitive closure of $R$ is denoted by $H$, and $x_{i}$ is said to be revealed preferred to $x_{s}$ if $x_{i} H x_{s}{ }^{3}$

Definition 2 The observations $\left(x_{t}, p_{t}\right)_{t=1, \ldots, n}$ satisfy the discrete axiom of revealed preference $(D A R P)$ if for any $i, j=1, \ldots, n$

$$
x_{i} H x_{j} \Rightarrow x_{i}+\mathbf{1} \notin B_{j}
$$

Now that DARP is formally defined, we can compare our approach with other recent ones, namely, Cosaert and Demuynck (2013) and Polisson and Quah (2013). As already mentioned in section 1.1, Cosaert and Demuynck (2013)'s data consist, at every date $t=1, \ldots, n$, of a choice among finitely many consumption bundles $\left\{b_{t}^{1}, \ldots, b_{t}^{N_{t}}\right\}$ with $b_{t}^{k} \in \mathbb{R}_{+}^{K}, k=1, \ldots, N_{t}$. Their framework is thus more general than ours since $B_{t} \cap \mathbb{N}^{K}$ is clearly finite. Their "weakly monotone axiom of revealed preference" (WMARP) can be formulated by first considering auxiliary budget sets $B_{t}^{\prime}=\left\{x \in \mathbb{R}_{+}^{K}: \exists b_{t}^{k}\right.$ such that $\left.x \leq b_{t}^{k}\right\}$. These budget sets are of course not finite nor linear, but one can still define a direct preference relation $R^{\prime}$ by $x_{i} R^{\prime} x_{j}$ if $x_{j} \in B_{j}^{\prime}$ and deduce its transitive closure $H^{\prime}$. WMARP then requires that $x_{i} R^{\prime} x_{j} \Rightarrow x_{i} \notin$ int $B_{j}^{\prime}$. It is readily checked that WMARP is just GARP for the general budgets $B_{t}^{\prime}$ (see Forges and Minelli, 2009) and that if, at every date $t$, the finite budget sets are generated by discrete linear budget sets $B_{t} \cap \mathbb{N}^{K}$, as in the current paper, WMARP and DARP are equivalent.

When WMARP holds, Cosaert and Demuynck (2013) construct a utility function over the whole $\mathbb{R}_{+}^{K}$, by adapting Afriat's methodology to nonlinear budget sets, exactly as in Forges and Minelli (2009). This utility function has no particular property beyond monotonicity. As will become clear in sections 4 and 6, our application of Afriat's methodology keeps track of

\footnotetext{
${ }^{3}$ That is, $x_{i} H x_{s}$ if there exists an ordered subset $\{i, j, k, \ldots, r, s\} \subset\{1, \ldots, n\}$ such that $x_{i} R x_{j}, x_{j} R x_{k}, \ldots$, $x_{r} R x_{s}$.
} 
the linear structure behind the discrete budget sets, which allows us to derive a quasi-concave utility function.

Polisson and Quah (2013) study to which extent the standard GARP, which is tailored for perfectly divisible goods (and linear budget sets), is still appropriate when goods are indivisible. Let us maintain the term GARP for the case of perfectly divisible goods, as in Polisson and Quah (2013), and compare GARP with DARP. On the one hand, the consistency condition of definition 2 can be rewritten: for any $i, j=1, \ldots, n, x_{i} H x_{j}$ implies $\left(\left\{x_{i}\right\}+\mathbb{N}_{++}^{K}\right) \cap B_{j}=\emptyset$. On the other hand, the observations satisfy GARP if for any $j, k=1, \ldots, n, x_{i} H x_{j}$ implies $p_{j} \cdot x_{i} \geq p_{j} \cdot x_{j}$, which can be rewritten: for any $i, j=1, \cdots, n, x_{i} H x_{j}$ implies $\left(\left\{x_{i}\right\}+\mathbb{R}_{++}^{K}\right) \cap B_{j}=\emptyset$. This shows that DARP and GARP express exactly the same consistency property, the only difference being discrete space versus continuous space. ${ }^{4}$ Thus it is tempting to say that DARP appears as the right version of GARP when goods are indivisible.

Adopting the viewpoint of Polisson and Quah (2013), one sees that the continuous axiom (GARP) is a stronger requirement than the discrete one (DARP). Observations consistent with DARP but not with GARP are given in figure 1, others that violate both DARP and GARP are given in figure 2 .

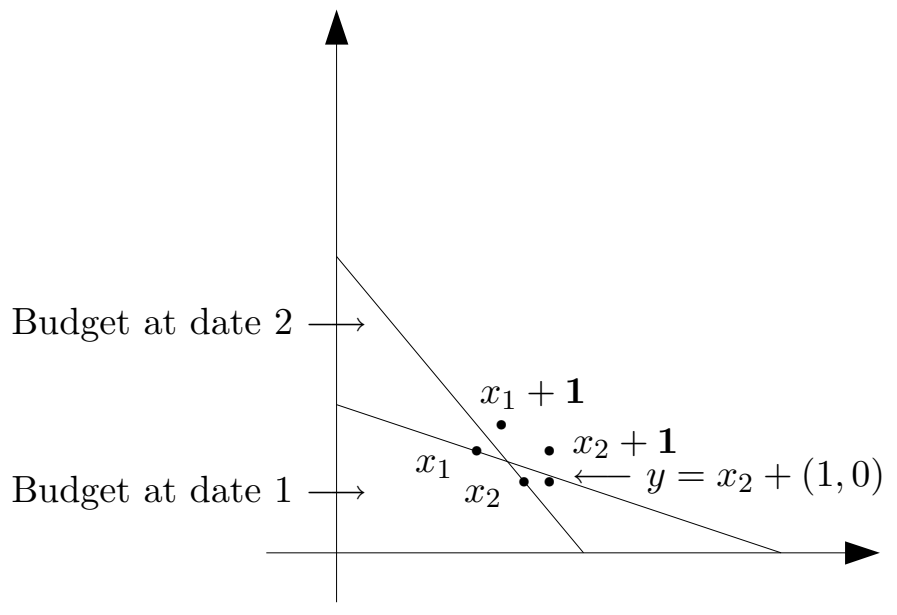

Figure 1: Passing DARP - Violation of GARP

\footnotetext{
${ }^{4}$ This also implies that DARP is computationally equivalent to GARP. It amounts to compute the transitive closure $H$, which can be done efficiently by using Warshall's algorithm (Varian, 1982).
} 


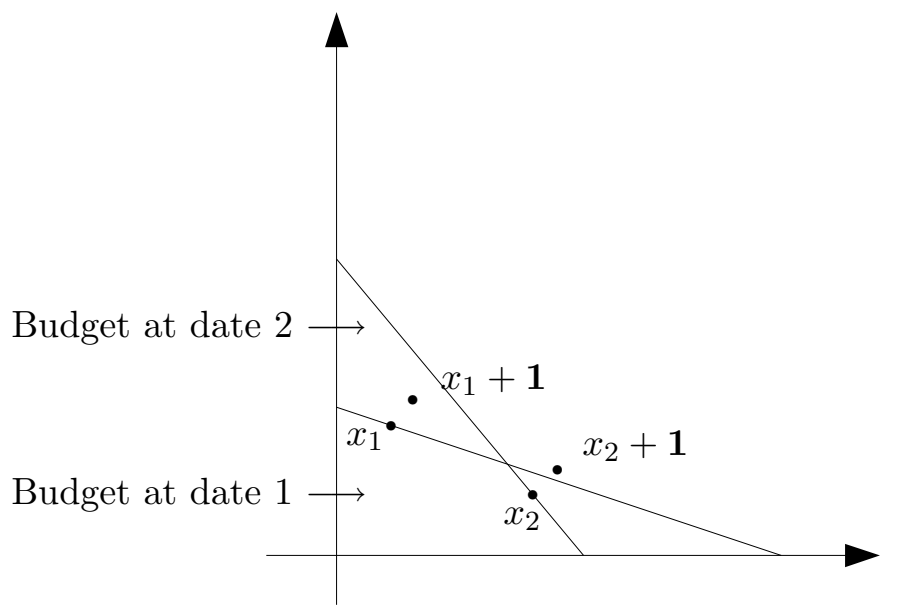

Figure 2: Violation of DARP (and GARP)

The previous discussion suggests that the reason which makes GARP, in its standard form, inappropriate to deal with indivisible goods, does not come from the indivisibilities themselves but rather from the nonlinearities that they generate. By making further assumptions on the model, Polisson and Quah (2013) nevertheless reconcile the standard GARP with indivisible goods.

In the situation depicted by figure 1, suppose that the consumer behaves according to Eq.(1), then he is necessarily indifferent between $x_{1}$ and $x_{2}$. It follows that he does not meet cost efficiency since $p_{1} \cdot x_{2}<p_{1} \cdot x_{1}$. Such a situation is excluded by allowing either for an implicit continuous good (Polisson and Quah, 2013) or for money (Brown and Calsamiglia, 2007; Sákovics, 2013), or by imposing cost efficiency (Fujishige and Yang, 2012). But, nothing prevents rationality as given by Eq.(1) only to coexist with cost inefficiencies if goods are available only in discrete quantities. In figure 1, the consumer has no way to take advantage from the monetary gain that results from choosing $x_{2}$ instead of $x_{1}$, at date 1 .

In what follows we will show that DARP is the appropriate condition for consumption data to be consistent with utility maximization, in the sense of Eq.(1), as long as all goods are traded in discrete quantities. 


\section{MAIN RESULTS}

The next proposition is our first main result.

Proposition 1 The observations $\left(x_{t}, p_{t}\right)_{t=1, \ldots, n}$ satisfy DARP if, and only if, there exists a discrete quasi-concave and monotonic rationalization of the observations.

One shortcoming of the result is that we do not obtain a strictly monotonic rationalization. The following example shows that, under DARP, there is no hope in general to get such a property.

Example Let $\left[x_{1}=(5,4) ; p_{1}=(1,3.2)\right]$ and $\left[x_{2}=(7,3) ; p_{2}=(3,2)\right]$ be the set of observations. The observations satisfy DARP and, by proposition 1 , there exists a monotonic rationalization $u$ which necessarily satisfies $u\left(x_{1}\right)=u\left(x_{2}\right)$ since $x_{1}$ and $x_{2}$ are both affordable at both dates. Note that $y:=x_{2}+(1,0)=(8,3)$ is also affordable at date 1 . Therefore, the utility function $u$ cannot be strictly monotonic, otherwise $u(y)>u\left(x_{2}\right)=u\left(x_{1}\right)$ would contradict that $x_{1}$ has been purchased at date 1 . Figure 1 illustrates this example.

Let us now define a stronger axiom than DARP to obtain strict monotonicity. Given the set of observations, let $c(t)$ be one of the cheapest goods at date $t$, that is, $c(t) \in \operatorname{argmin}\left\{p_{t}^{g}: g=\right.$ $1, \ldots, K\}$.

Definition 3 The observations $\left(x_{t}, p_{t}\right)_{t=1, \ldots, n}$ satisfy $D A R P^{*}$ if for any $i, j=1, \ldots, n$

$$
x_{i} H x_{j} \Rightarrow x_{i}+\mathbf{e}^{c(j)} \notin B_{j}
$$

In figure 1 , the bundle $y$ is affordable at date 1 , hence the set of observations does not pass DARP*. Using DARP* we obtain an analog to proposition 1 with the additional property of strict monotonicity.

Proposition 2 The observations $\left(x_{t}, p_{t}\right)_{t=1, \ldots, n}$ satisfy DARP* if, and only if, there exists a discrete quasi-concave and strictly monotonic rationalization of the observations.

To see why DARP and DARP* refer to one or the other version of monotonicity observe simply that DARP* amounts to: for any $i, j=1, \ldots, n, x_{i} H x_{j}$ implies $\left(\left\{x_{i}\right\}+\mathbb{N}^{K} \backslash\{0\}\right) \cap B_{j}=\emptyset$, while DARP requires $\left(\left\{x_{i}\right\}+\mathbb{N}_{++}^{K}\right) \cap B_{j}=\emptyset .^{5}$

\footnotetext{
${ }^{5}$ Such a distinction has not bite if one considers GARP, as long as prices are positive, since $\left(\left\{x_{i}\right\}+\mathbb{R}_{++}^{K}\right) \cap B_{j}=\emptyset$ is equivalent to $\left(\left\{x_{i}\right\}+\mathbb{R}_{+}^{K} \backslash\{0\}\right) \cap B_{j}=\emptyset$. In other words, one gets strict monotonicity for free in the continuous case.
} 
Finally, it is worth pointing out that DARP and DARP* can be restated in terms of an operational property of cyclical consistency, as it is also the case for GARP.

Definition 4 An $n \times n$ real matrix $A=\left(a_{j k}\right)_{j, k=1, \ldots, n}$ is cyclically consistent if for any ordered subset $\{j, k, \ell, \ldots, r\} \subset\{1, \ldots, n\}, a_{j k} \leq 0, a_{k \ell} \leq 0, \ldots, a_{r j} \leq 0$ implies all terms are 0 .

Given the observations $\left(x_{t}, p_{t}\right)_{t=1, \ldots, n}$, let $\boldsymbol{\beta}=\left(\beta_{j k}\right)_{j, k=1, \ldots, n}$ be the $n \times n$ matrix where: $\beta_{j k} \in\{-1,0,+1\} ; \beta_{j k} \leq 0$ if $x_{k} \in B_{j}$, with strict inequality iff $x_{k}+\mathbf{1} \in B_{j} ; \beta_{j k}=+1$ if $x_{k} \notin B_{j}$. Let $\tilde{\boldsymbol{\beta}}$ be the matrix where $\tilde{\beta}_{j k} \in\{-1,0,+1\} ; \tilde{\beta}_{j k} \leq 0$ if $x_{k} \in B_{j}$, with strict inequality iff $x_{k}+\mathbf{e}^{c(j)} \in B_{j} ; \tilde{\beta}_{j k}=+1$ if $x_{k} \notin B_{j}$.

The next proposition can be shown by using standard arguments (see, e.g., Forges and Minelli, 2009, p.138). Its proof is thus omitted.

Proposition 3 The observations satisfy DARP (resp. DARP*) if, and only if, the matrix $\boldsymbol{\beta}$ (resp. $\tilde{\boldsymbol{\beta}}$ ) is cyclically consistent. ${ }^{6}$

\section{About the proof of Propositions 1 And 2}

An interesting (and perhaps unexpected) feature of the proof of our main results (propositions 1 and 2) is that the construction of an explicit utility function from Afriat's inequalities goes through in our discrete framework.

For instance, showing that DARP is sufficient for the existence of a rationalization can be sketched as follows. By proposition 3, we know that DARP is equivalent to cyclical consistency of a matrix $\boldsymbol{\beta}$ with elements in $\{-1,0,1\}$ only. In addition, cyclical consistency is preserved by considering any matrix having entries with identical signs and zeros. We exploit this degree of freedom to deduce the existence of a solution for adequately chosen Afriat's inequalities. We obtain then $\psi_{1}, \ldots, \psi_{n}$ and $\delta_{1}, \ldots, \delta_{n}>0$ such that $u: \mathbb{N}^{K} \rightarrow \mathbb{R}$ defined as follows is a well-behaved rationalization of the observations:

$$
u(x)=\min \left\{\psi_{1}+\delta_{1} p_{1} \cdot\left(x-x_{1}\right) \mathbb{1}_{A_{1}^{c}}(x), \ldots, \psi_{n}+\delta_{n} p_{n} \cdot\left(x-x_{n}\right) \mathbb{1}_{A_{n}^{c}}(x)\right\}
$$

where $A_{t}=\left\{x \in \mathbb{N}^{K}: p_{t} \cdot\left(x_{t}-\mathbf{1}\right)<p_{t} \cdot x \leq p_{t} \cdot x_{t}\right\}$.

\footnotetext{
${ }^{6}$ The formulation in terms of a (qualitative) data matrix, which summarizes the affordability of the consumption bundles, is not typical to the discrete consumption space. In the continuous case, Forges and Minelli (2009), Ekeland and Galichon (2012) and Forges and Iehlé (2012) already provide insights on the formulation of GARP in terms of a data matrix. For GARP the entries of the matrix $\boldsymbol{\alpha}$ are $\alpha_{j k} \in\{-1,0,+1\} ; \alpha_{j k} \leq 0$ if $x_{k} \in B_{j}$, with strict inequality iff $p_{j} \cdot x_{k}<p_{j} \cdot x_{j}$; and $\alpha_{j k}=+1$ if $x_{k} \notin B_{j}$.
} 
A potential difficulty with our construction is that the desirable properties of a utility function are not a priori granted here, contrary to the standard competitive case where the utility function can be taken as the infimum of linear and increasing functions. Especially, the functions in the arguments of $u$ would be flat on a small domain if they were defined over a continuous consumption set. However we show that the restriction of $u$ to integer bundles is well-behaved, in particular monotonic and discrete quasi-concave. ${ }^{7}$

To establish proposition 2, we can proceed identically by using the entries of the data matrix $\tilde{\boldsymbol{\beta}}$. In that case, we get in addition that the resulting utility function is then strictly monotonic.

\section{Non BINDING BUdGET CONSTRAints}

Under monotonic preferences the rational consumer holds an unobserved revenue $r_{t}$ at date $t$ such that $p_{t} \cdot x_{t} \leq r_{t}$ and $p_{t} \cdot x>r_{t}$ for every $x \gg x_{t}$, with $x \in \mathbb{N}^{K}$. Contrary to the perfectly divisible case, this does not imply in our framework that $p_{t} \cdot x_{t}=r_{t}{ }^{8}$ Indeed, the consumer is a priori not able (or not willing) to exhaust fully his revenue if the remaining amount after purchasing $x_{t}$ leaves no room to buy one additional unit of each good. It follows that, instead of $B_{t}$, the analyst may be willing to consider larger budget sets, which are compatible with such typical losses.

Our previous results are virtually not affected by allowing for such budgets. We parametrize the budget sets that are compatible with the data through budget gaps $\boldsymbol{\theta}$. Formally, a family of budget gap parameters is $\boldsymbol{\theta}=\left(\theta_{t}\right)_{t=1, \ldots, n}$, with $\theta_{t} \in[0,1) \cdot{ }^{9}$ Given $\boldsymbol{\theta}$, the budget at date $t$ is:

$$
B_{t}^{\boldsymbol{\theta}}:=\left\{x \in \mathbb{R}_{+}^{K}: p_{t} \cdot x \leq p_{t} \cdot\left(x_{t}+\theta_{t} \mathbf{1}\right)\right\}
$$

The polar case that was defined at the beginning of the section corresponds to $\boldsymbol{\theta}=0 .{ }^{10}$

A utility function $u: \mathbb{N}^{K} \rightarrow \mathbb{R}$ is called a $\boldsymbol{\theta}$-rationalization of the observations $\left(x_{t}, p_{t}\right)_{t=1, \ldots, n}$ if, at each date $t, x_{t}$ solves $\max u(x)$ subject to $x \in B_{t}^{\boldsymbol{\theta}} \cap \mathbb{N}^{K}$. Let $R^{\boldsymbol{\theta}}$ be the direct revealed preference relation: $x_{i} R^{\boldsymbol{\theta}} x_{j}$, if $x_{j} \in B_{i}^{\boldsymbol{\theta}}$. Let $H^{\boldsymbol{\theta}}$ be the transitive closure of $R^{\boldsymbol{\theta}}$. The observations satisfies $\boldsymbol{\theta}$-DARP if for any $i, j=1, \cdots, n, x_{i} H^{\boldsymbol{\theta}} x_{j}$ implies $x_{i}+\mathbf{1} \notin B_{j}^{\boldsymbol{\theta}}$.

\footnotetext{
${ }^{7}$ In the competitive case, the function can be chosen as follows: $\bar{u}(x)=\min \left\{\bar{\psi}_{1}+\bar{\delta}_{1} p_{1} \cdot\left(x-x_{1}\right), \ldots, \bar{\psi}_{n}+\bar{\delta}_{n} p_{n}\right.$. $\left.\left(x-x_{n}\right)\right\}$ for some $\bar{\psi}_{1}, \ldots, \bar{\psi}_{n}$ and $\bar{\delta}_{1}, \ldots, \bar{\delta}_{n}>0$. In the case of finite choice sets, Cosaert and Demuynck (2013) use a different strategy to construct the rationalization, in our setting it amounts to consider the following mapping: $\underline{u}(x)=\min \left\{\underline{\psi}_{1}+\underline{\delta}_{1} \min _{y_{1} \in B_{1} \cap \mathbb{N} K} \max _{i=1, \ldots, K} e^{i} \cdot\left(x-y_{1}\right), \ldots, \underline{\psi}_{n}+\underline{\delta}_{n} \min _{y_{n} \in B_{n} \cap \mathbb{N} K} \max _{i=1, \ldots, K} e^{i} \cdot\left(x-y_{n}\right)\right\}$ for some $\underline{\psi}_{1}, \ldots, \underline{\psi}_{n}$ and $\underline{\delta}_{1}, \ldots, \underline{\delta}_{n}>0$, which is not quasi-concave in general.

${ }^{8}$ Actually, one needs at least one perfectly divisible and desirable good to obtain the equality.

${ }^{9}$ The limit case $\theta_{t}=1$ is treated separately in section 6.3.

${ }^{10}$ Echenique et al. (2011b) also acknowledge the possibility of non binding budget constraints in the indivisible case. A key difference in their model is that the true revenue at each date is part of the observations.
} 
Proposition 4 For any family of budget gaps $\boldsymbol{\theta}=\left(\theta_{t}\right)_{t=1, \ldots, n}$, with $\theta_{t} \in[0,1)$, the observations $\left(x_{t}, p_{t}\right)_{t=1, \ldots, n}$ satisfy $\boldsymbol{\theta}$-DARP if, and only if, there exists a discrete quasi-concave and monotonic $\boldsymbol{\theta}$-rationalization of the observations.

As before we obtain an analogous result for the strictly monotonic case. Given $\boldsymbol{\theta}$, the budget set at date $t$ is:

$$
\widetilde{B}_{t}^{\boldsymbol{\theta}}:=\left\{x \in \mathbb{R}_{+}^{K}: p_{t} \cdot x \leq p_{t} \cdot\left(x_{t}+\theta_{t} \mathbf{e}^{c(t)}\right)\right\}
$$

A utility function $u: \mathbb{N}^{K} \rightarrow \mathbb{R}$ is called a strong $\boldsymbol{\theta}$-rationalization of the observations $\left(x_{t}, p_{t}\right)_{t=1, \ldots, n}$ if, at each date $t, x_{t}$ solves $\max u(x)$ subject to $x \in \widetilde{B}_{t}^{\boldsymbol{\theta}} \cap \mathbb{N}^{K}$. Let $\widetilde{R}^{\boldsymbol{\theta}}$ be the direct revealed preference relation: $x_{i} \widetilde{R}^{\boldsymbol{\theta}} x_{j}$, if $x_{j} \in \widetilde{B}_{i}^{\boldsymbol{\theta}}$. Let $\widetilde{H}^{\boldsymbol{\theta}}$ be the transitive closure of $\widetilde{R}^{\boldsymbol{\theta}}$. The observations satisfies $\boldsymbol{\theta}$-DARP ${ }^{*}$ if for any $i, j=1, \cdots, n, x_{i} \widetilde{H}^{\boldsymbol{\theta}} x_{j}$ implies $x_{i}+\mathbf{e}^{c(j)} \notin \widetilde{B}_{j}^{\boldsymbol{\theta}}$.

Proposition 5 For any family of budget gaps $\boldsymbol{\theta}=\left(\theta_{t}\right)_{t=1, \ldots, n}$, with $\theta_{t} \in[0,1)$, the observations $\left(x_{t}, p_{t}\right)_{t=1, \ldots, n}$ satisfy $\boldsymbol{\theta}$-DARP* if, and only if, there exists a discrete quasi-concave and strictly monotonic strong- $\boldsymbol{\theta}$-rationalization of the observations.

Remark 1 Observe first that $B_{t}^{\boldsymbol{\theta}} \subseteq B_{t}^{\boldsymbol{\theta}^{\prime}}$ and $\widetilde{B}_{t}^{\boldsymbol{\theta}} \subseteq \widetilde{B}_{t}^{\boldsymbol{\theta}^{\prime}}$ if $\theta_{t} \leq \theta_{t}^{\prime}$. Hence the larger the gaps, the more demanding the parametrized axiom. In practice, this suggests a possible strategy for the analyst, who is facing a range of rationalization tests. If DARP is satisfied by the data $(\boldsymbol{\theta}=0)$, the analyst may look for larger and larger budget sets that are consistent with rationalization by conducting successive tests where parameters $\boldsymbol{\theta}$ are incremented step by step.

Remark 2 The previous remark also motivates the definition of the largest budget set at each date, which is as follows: $B_{t}^{1}:=\left\{x \in \mathbb{R}_{+}^{K}: p_{t} \cdot x<p_{t} \cdot\left(x_{t}+\mathbf{1}\right)\right\}$. One deduces immediately the corresponding definitions of rationalization and DARP, say 1-rationalization and 1-DARP. The conclusion of proposition 4 (and 5) can be easily extended to that case (see Section 6.3). In addition, the exercise features an interesting property. Indeed, from a quick look at the definition of 1-DARP we derive that 1-DARP is a stronger requirement than GARP. Hence GARP stands between DARP and 1-DARP.

Remark 3 Adjusting budget sets according to a family of parameters is also the method to define Afriat's critical cost efficiency index and its variants (see, e.g., Varian, 1990). Such smaller and fictitious budgets are constructed to measure how close the data are to satisfying standard GARP. Despite an apparent analogy our parametrization has a complete different purpose and actually operates the other way around by increasing the available budgets. 
Remark 4 Our analysis concentrates on the standard consumer model with linear budgets. However budget sets may display nonconvexities in practice. In the light of our discussion in section 2, we note that the approach of Cosaert and Demuynck (2013) would apply in that case and in presence of indivisibilities. More precisely, their methodology is well designed to test the rationality of a consumer maximizing his utility over $B_{t} \cap \mathbb{N}^{K}$ at each date $t$, where $\left(B_{t}\right)_{t}$ is now defined as a family of comprehensive and compact subsets of $\mathbb{R}_{+}^{K}$, as in Forges and Minelli (2009).

\section{APPENDIX}

In what follows we demonstrate propositions 4 and 5 (proposition 1 (resp. 2) corresponds to the polar case $\boldsymbol{\theta}=0$ in proposition 4 (resp. 5)).

\subsection{Proof of proposition 4}

Given the observations $\left(x_{t}, p_{t}\right)_{t=1, \ldots, n}$ and a family of budget gaps $\boldsymbol{\theta}=\left(\theta_{t}\right)_{t=1, \ldots, n}, \theta_{t} \in[0,1)$, let $\boldsymbol{\beta}^{\boldsymbol{\theta}}=\left(\beta_{j k}\left(\theta_{j}\right)\right)_{j, k=1, \ldots, n}$ be an $n \times n$ matrix where:

$$
\begin{aligned}
& \beta_{j k}\left(\theta_{j}\right) \in\{-1,0,+1\} \\
& \beta_{j k}\left(\theta_{j}\right) \leq 0 \text { if } p_{j} \cdot x_{k} \leq p_{j} \cdot\left(x_{j}+\theta_{j} \mathbf{1}\right) ; \text { with strict inequality iff } p_{j} \cdot\left(x_{k}+\mathbf{1}\right) \leq p_{j} \cdot\left(x_{j}+\theta_{j} \mathbf{1}\right) \\
& \beta_{j k}\left(\theta_{j}\right)=+1 \text { if } p_{j} \cdot x_{k}>p_{j} \cdot\left(x_{j}+\theta_{j} \mathbf{1}\right) .
\end{aligned}
$$

Observe first that the analog of proposition 3 holds true, that is, the observations $\left(x_{t}, p_{t}\right)_{t=1, \ldots, n}$ satisfy $\boldsymbol{\theta}$-DARP iff $\boldsymbol{\beta}^{\boldsymbol{\theta}}$ is cyclically consistent. In the remainder we will refer to the latter property as $\boldsymbol{\theta}$-DARP.

We first show that $\boldsymbol{\theta}$-DARP is necessary for utility maximization. Let $u$ be a monotonic $\boldsymbol{\theta}$-rationalization of the observations $\left(x_{t}, p_{t}\right)_{t=1, \ldots, n}$ and $\{j, k, \ell, \ldots, r\}$ be an ordered subset of $\{1, \ldots, n\}$ such that $\beta_{j k}\left(\theta_{j}\right) \leq 0, \beta_{k \ell}\left(\theta_{k}\right) \leq 0, \ldots, \beta_{r j}\left(\theta_{r}\right) \leq 0$. For any two consecutive elements $a, b \in\{j, k, \ell, \ldots, r\}, u\left(x_{a}\right) \geq u\left(x_{b}\right)$. The entire sequence of inequalities implies that $u\left(x_{a}\right)=$ $u\left(x_{b}\right)$ for every $a, b \in\{j, k, \ell, \ldots, r\}$. By construction, if $\beta_{a b}\left(\theta_{a}\right)<0$ for two consecutive elements $a, b \in\{j, k, \ell, \ldots, r\}$ then $x_{b}+\mathbf{1} \in B_{a}^{\boldsymbol{\theta}}$ and $u\left(x_{b}+\mathbf{1}\right)>u\left(x_{b}\right)=u\left(x_{a}\right)$ since $u$ is monotonic. It contradicts that $x_{a}$ has been purchased at date $a$. Hence $\boldsymbol{\theta}$-DARP is satisfied.

We show now the converse implication. Suppose that $\left(x_{t}, p_{t}\right)_{t=1, \ldots, n}$ satisfies $\boldsymbol{\theta}$-DARP and define a new data matrix $\boldsymbol{\gamma}^{\boldsymbol{\theta}}$, with entries $\gamma_{j k}\left(\theta_{j}\right)=\left|\beta_{j k}\left(\theta_{j}\right)\right| p_{j} \cdot\left(x_{k}-\left(x_{j}+\theta_{j} \mathbf{1}\right)\right)$.

By assumption, $\boldsymbol{\beta}^{\boldsymbol{\theta}}$ satisfies cyclical consistency. It is an easy matter to verify that it is also the case for the matrix $\gamma^{\boldsymbol{\theta}}$ since, in particular, $\gamma_{j k}\left(\theta_{j}\right) \leq 0$ iff $p_{j} \cdot\left(x_{k}-\left(x_{j}+\theta_{j} \mathbf{1}\right)\right) \leq 0$ iff 
$\beta_{j k}\left(\theta_{j}\right) \leq 0$. We can obtain therefore the following Afriat's inequalities (see, e.g., Fostel et al., 2004). ${ }^{11}$ There exist $\psi_{1}, \ldots, \psi_{n}$ and $\delta_{1}, \ldots, \delta_{n}>0$ such that

$$
\psi_{k} \leq \psi_{j}+\delta_{j} \gamma_{j k}\left(\theta_{j}\right) \quad \forall j, k=1, \ldots, n
$$

For every $t=1, \ldots, n$, define

$$
A_{t}=\left\{x \in \mathbb{N}^{K}: p_{t} \cdot\left(x_{t}-\left(1-\theta_{t}\right) \mathbf{1}\right)<p_{t} \cdot x \leq p_{t} \cdot\left(x_{t}+\theta_{t} \mathbf{1}\right)\right\}
$$

Let $u: \mathbb{N}^{K} \rightarrow \mathbb{R}$ be defined as follows:

$$
u(x)=\min \left\{\psi_{1}+\delta_{1} p_{1} \cdot\left(x-\left(x_{1}+\theta_{1} \mathbf{1}\right)\right) \mathbb{1}_{A_{1}^{c}}(x), \ldots, \psi_{n}+\delta_{n} p_{n} \cdot\left(x-\left(x_{n}+\theta_{n} \mathbf{1}\right)\right) \mathbb{1}_{A_{n}^{c}}(x)\right\}
$$

Let us show that the function $u$ rationalizes the experiment. From the definition of $\boldsymbol{\beta}$, it holds, for any $t=1, \ldots, n$, that $u\left(x_{t}\right)=\min \left\{\psi_{1}+\delta_{1} p_{1} \cdot\left(x_{t}-\left(x_{1}+\theta_{1} \mathbf{1}\right)\right) \mathbb{1}_{A_{1}^{c}}\left(x_{t}\right), \ldots, \psi_{n}+\delta_{n} p_{n}\right.$. $\left.\left(x_{t}-\left(x_{n}+\theta_{n} \mathbf{1}\right)\right) \mathbb{1}_{A_{n}^{c}}\left(x_{t}\right)\right\}=\min \left\{\psi_{1}+\delta_{1} p_{1} \cdot\left(x_{t}-\left(x_{1}+\theta_{1} \mathbf{1}\right)\right)\left|\beta_{1 t}\left(\theta_{1}\right)\right|, \ldots, \psi_{n}+\delta_{n} p_{n} \cdot\left(x_{t}-\left(x_{n}+\right.\right.\right.$ $\left.\left.\left.\theta_{n} \mathbf{1}\right)\right)\left|\beta_{n t}\left(\theta_{n}\right)\right|\right\}=\min \left\{\psi_{1}+\delta_{1} \gamma_{1 t}\left(\theta_{1}\right), \ldots, \psi_{n}+\delta_{n} \gamma_{n t}\left(\theta_{n}\right)\right\}$.

Since $\gamma_{t t}\left(\theta_{t}\right)=0$, from the Afriat's inequalities, Eq. $(*)$, we get that $u\left(x_{t}\right)=\psi_{t}$.

Next, consider $x \in X$ such that $p_{t} \cdot x \leq p_{t} \cdot\left(x_{t}+\theta_{t} \mathbf{1}\right)$, then

$$
\begin{aligned}
u(x) & =\min \left\{\psi_{1}+\delta_{1} p_{1} \cdot\left(x-\left(x_{1}+\theta_{1} \mathbf{1}\right)\right) \mathbb{1}_{A_{1}^{c}}(x), \ldots, \psi_{n}+\delta_{n} p_{n} \cdot\left(x-\left(x_{n}+\theta_{n} \mathbf{1}\right)\right) \mathbb{1}_{A_{n}^{c}}(x)\right\} \\
& \leq \psi_{t}+\delta_{t} p_{t} \cdot\left(x-\left(x_{t}+\theta_{t} \mathbf{1}\right)\right) \mathbb{1}_{A_{t}^{c}}(x) \\
& \leq \psi_{t}=u\left(x_{t}\right)
\end{aligned}
$$

(by using $\delta_{t}, \mathbb{1}_{A_{t}}(x) \geq 0$ and $\left.p_{t} \cdot\left(x-\left(x_{t}+\theta_{t} \mathbf{1}\right)\right) \leq 0\right)$

Hence $u$ rationalizes the data. Clearly the function is monotonic (but not strictly monotonic!). The next lemma concludes the proof of proposition 4.

Lemma 1 The function $u$ is discrete quasi-concave.

Proof To make use of customary arguments, let us assume first that the mapping $u$, constructed before, is defined on $X=\mathbb{R}_{+}^{K}$ instead of $\mathbb{N}^{K}$ (idem for the sets $A_{t}, t=1, \ldots, n$ ). We prove that $u$ is quasi-concave. Note that quasi-concavity is invariant to pointwise infimum of quasi-concave functions. Hence it suffices to prove quasi-concavity for the functions

$$
f_{t}(x):=p_{t} \cdot\left(x-\left(x_{t}+\theta_{t} \mathbf{1}\right)\right) \mathbb{1}_{A_{t}}(x), \quad t=1, \ldots, n
$$

It amounts to show that for each $x, x^{\prime} \in X$ and $\lambda \geq 0, f_{t}\left(\lambda x+(1-\lambda) x^{\prime}\right) \geq \min \left\{f_{t}(x), f_{t}\left(x^{\prime}\right)\right\}$. Let $X_{1}, X_{2}, X_{3} \in X$ be three disjoint sets defined as follows: $X_{1}=\left\{x \in X: p_{t} \cdot\left(x-\left(x_{t}-\left(1-\theta_{t}\right) \mathbf{1}\right)\right) \leq\right.$

\footnotetext{
${ }^{11}$ The existence of a solution to the Afriat's inequalities relies only on the cyclical consistency of the matrix.
} 
$0\}, X_{2}=A_{t}(x), X_{3}=\left\{x \in X: p_{t} \cdot\left(x-\left(x_{t}+\theta_{t} \mathbf{1}\right)\right)>0\right\}$. It is an easy matter to verify that $X_{1}, X_{2}, X_{3}, X_{1} \cup X_{2}, X_{2} \cup X_{3}$ are all convex sets (note also that $X=\cup_{i=1, \ldots, 3} X_{i}$ ). We need to consider several cases.

If $x, x^{\prime} \in X_{i}$, for some $i=1, \ldots 3$, then $\lambda x+(1-\lambda) x^{\prime} \in X_{i}$ and the required inequality is verified since either $f_{t}=0$ (case $X_{2}$ ) or $f_{t}$ is linear (cases $X_{1}$ and $X_{3}$ ). If $x \in X_{2}$ and $x^{\prime} \in X_{3}$, then $\lambda x+(1-\lambda) x^{\prime} \in X_{2} \cup X_{3}$; it follows that $f_{t}\left(x^{\prime}\right) \geq f_{t}\left(\lambda x+(1-\lambda) x^{\prime}\right) \geq f_{t}(x)=0$ (either $f_{t}\left(\lambda x+(1-\lambda) x^{\prime}\right)=0$ if $\lambda x+(1-\lambda) x^{\prime} \in X_{2}$ or $f_{t}\left(\lambda x+(1-\lambda) x^{\prime}\right)=(1-\lambda) p_{t} \cdot\left(x^{\prime}-\left(x_{t}+\theta_{t} \mathbf{1}\right)\right)>0$ if $\left.\lambda x+(1-\lambda) x^{\prime} \in X_{3}\right)$. The same reasoning applies to the case $x \in X_{2}$ and $x^{\prime} \in X_{1}$. Finally, it remains to consider the case $x \in X_{1}$ and $x^{\prime} \in X_{3}$. Either $\lambda x+(1-\lambda) x^{\prime} \in X_{1} \cup X_{3}$ and then $f_{t}\left(\lambda x+(1-\lambda) x^{\prime}\right)=\lambda p_{t} \cdot\left(x-\left(x_{t}+\theta_{t} \mathbf{1}\right)\right)+(1-\lambda) p_{t} \cdot\left(x^{\prime}-\left(x_{t}+\theta_{t} \mathbf{1}\right)\right) \geq f_{t}(x)$ since $f_{t}(x)<f_{t}\left(x^{\prime}\right)$; or $\lambda x+(1-\lambda) x^{\prime} \in X_{2}$, and then $f_{t}\left(\lambda x+(1-\lambda) x^{\prime}\right)=0 \geq f_{t}(x)$ since $x \in X_{1}$ implies $p_{t} \cdot\left(x+\mathbf{1}-\left(x_{t}+\theta_{t} \mathbf{1}\right)\right) \leq 0$ which implies in turn $f_{t}(x) \leq 0$.

Hence $u$ is quasi-concave on $\mathbb{R}_{+}^{K}$. Clearly, the restriction of $u$ to $\mathbb{N}^{K}$ is a fortiori discrete quasi-concave.

\subsection{Proof of proposition 5}

The proof is readily analogous to the one of proposition 4 and amounts to replacing the vector $\mathbf{1}$ by $\mathbf{e}^{c(t)}$ at each date $t$. We omit that tedious repetition. However let us show explicitly how we obtain a rationalization that is strictly monotonic, in the only if part of the proof.

Following the arguments of the proof of proposition 4, one is led to the following construction of the rationalization :

$$
\tilde{u}(x)=\min \left\{\tilde{\psi}_{1}+\tilde{\delta}_{1} \tilde{f}_{1}(x) \ldots, \tilde{\psi}_{n}+\tilde{\delta}_{n} \tilde{f}_{n}(x)\right\}
$$

where, for every $t=1, \ldots, n$,

$$
\begin{gathered}
\tilde{\psi}_{t} \geq 0, \tilde{\delta}_{t}>0 \\
\tilde{f}_{t}(x):=\left[p_{t} \cdot x-\left(p_{t} \cdot x_{t}+\theta_{t} p_{t}^{c(t)}\right)\right] \mathbb{1}_{\tilde{A}_{t}^{c}}(x)
\end{gathered}
$$

and

$$
\tilde{A}_{t}:=\left\{x \in \mathbb{N}^{K}: p_{t} \cdot x_{t}-\left(1-\theta_{t}\right) p_{t}^{c(t)}<p_{t} \cdot x \leq p_{t} \cdot x_{t}+\theta_{t} p_{t}^{c(t)}\right\}
$$

To verify strict monotonicity, it suffices to prove it for the functions $\tilde{f}_{t}(x), t=1, \ldots, n$. Suppose that $x \in \tilde{A}_{t}^{c}$ and $x^{\prime} \supsetneqq x$. If $x^{\prime} \in \tilde{A}_{t}^{c}$ then clearly $\tilde{f}_{t}\left(x^{\prime}\right)=p_{t} \cdot\left(x^{\prime}-x_{t}\right)-\theta_{t} p_{t}^{c(t)}>$ $p_{t} \cdot\left(x-x_{t}\right)-\theta_{t} p_{t}^{c(t)}=\tilde{f}_{t}(x)$ since prices are positive. If $x^{\prime} \in \tilde{A}_{t}$ it must be the case that $x$ belongs to the lower part of $\tilde{A}_{t}^{c}$, i.e. $p_{t} \cdot x \leq p_{t} \cdot x_{t}-\left(1-\theta_{t}\right) p_{t}^{c(t)}$, thus $\tilde{f}_{t}\left(x^{\prime}\right)=0>p_{t} \cdot\left(x-x_{t}\right)-\theta_{t} p_{t}^{c(t)}=f_{t}(x)$. Suppose that $x \in \tilde{A}_{t}$ and $x^{\prime} \supsetneqq x$, then it follows that $p_{t} \cdot x^{\prime} \geq p_{t} \cdot x+p_{t}^{c(t)}$. Since $x \in \tilde{A}_{t}$, we get 
that $p_{t} \cdot x^{\prime}>p_{t} \cdot x_{t}-\left(1-\theta_{t}\right) p_{t}^{c(t)}+p_{t}^{c(t)}$, that is, $p_{t} \cdot x^{\prime}>p_{t} \cdot x_{t}+\theta_{t} p_{t}^{c(t)}$. Hence we have just shown that $\tilde{f}_{t}\left(x^{\prime}\right)>0$. Since $\tilde{f}_{t}(x)=0$, the result is proved.

\subsection{The limit case for budget gaps}

We provide here a formal account to remark 2. Consider the following budget set at each date $t$ :

$$
B_{t}^{1}:=\left\{x \in \mathbb{R}_{+}^{K}: p_{t} \cdot x<p_{t} \cdot\left(x_{t}+\mathbf{1}\right)\right\}
$$

A utility function $u: \mathbb{N}^{K} \rightarrow \mathbb{R}$ is called a 1-rationalization of the observations if, at each date $t, x_{t}$ solves Eq.(1), with budgets $\left(B_{t}^{\mathbf{1}}\right)_{t}\left(\right.$ instead of $\left.\left(B_{t}\right)_{t}\right)$. Let $R^{\mathbf{1}}$ be the direct revealed preference relation defined according to budgets $\left(B_{t}^{\mathbf{1}}\right)_{t}$ and $H^{\mathbf{1}}$ be its transitive closure. DARP can be then restated as follows: the observations $\left(x_{t}, p_{t}\right)_{t=1, \ldots, n}$ satisfy 1 -DARP if for any $i, j=1, \ldots, n$, $x_{i} H^{\mathbf{1}} x_{j}$ implies $p_{j} \cdot x_{i} \geq p_{j} \cdot x_{j}$.

Note that 1-DARP comes close to the standard formulation of GARP. The difference relies only in the definition of the relation $H^{\mathbf{1}}$, which accounts for the largest budget sets. We obtain immediately that GARP is implied by 1 -DARP.

Observe that if $\theta_{t}$ is sufficiently close to 1 , with $t=1, \ldots, n$, a $\boldsymbol{\theta}$-rationalization is a $\mathbf{1}$ rationalization. In a similar way, we also get that if the observations satisfy $\boldsymbol{\theta}$-DARP for the $\theta$ s sufficiently close to 1 then the observations also satisfy 1-DARP. Since proposition 4 holds for every family of parameters $\boldsymbol{\theta}$ such that $\theta_{t} \in[0,1)$ we obtain the following corollary.

Corollary 1 The observations $\left(x_{t}, p_{t}\right)_{t=1, \ldots, n}$ satisfy 1 -DARP if, and only if, there exists a discrete quasi-concave and monotonic 1-rationalization of the observations.

We obtain an analogous corollary for the strictly monotonic case. The budget set at date $t$ is:

$$
\widetilde{B}_{t}^{1}:=\left\{x \in \mathbb{R}_{+}^{K}: p_{t} \cdot x<p_{t} \cdot\left(x_{t}+\mathbf{e}^{c(t)}\right)\right\}
$$

A utility function $u: \mathbb{N}^{K} \rightarrow \mathbb{R}$ is called a strong-1-rationalization of the observations if, at each date $t, x_{t}$ solves Eq.(1) with budgets $\left(\widetilde{B}_{t}^{\mathbf{1}}\right)_{t}$. Let $\widetilde{R}^{\mathbf{1}}$ be the direct revealed preference relation defined according to budgets $\left(\widetilde{B}_{t}^{1}\right)_{t}$, and $\widetilde{H}^{\mathbf{1}}$ be its transitive closure. The observations $\left(x_{t}, p_{t}\right)_{t=1, \ldots, n}$ satisfy $\mathbf{1 - D A R P} *$ if for any $i, j=1, \ldots, n, x_{i} \widetilde{H}^{\mathbf{1}} x_{j}$ implies $p_{j} \cdot x_{i} \geq p_{j} \cdot x_{j}$.

Corollary 2 The observations $\left(x_{t}, p_{t}\right)_{t=1, \ldots, n}$ satisfy 1 -DARP* if, and only if, there exists a discrete quasi-concave and strictly monotonic strong 1-rationalization of the observations. 
We omit the similar treatment of closely related congurations where the polar case $\theta_{t}=1$ are considered only at some dates $t=1, \ldots, n$. The resulting corollaries would be essentially the same.

\section{REFERENCES}

Afriat, S. (1967). The construction of a utility function from demand data. International Economic Review, 8, 67-77.

Brown, D. and Calsamiglia, C. (2007). The nonparametric approach to applied welfare analysis. Economic Theory, 31(1), 183-188.

Chambers, C. and Echenique, F. (2009). Supermodularity and preferences. Journal of Economic Theory, 144(3), 1004-1014.

Cosaert, S. and Demuynck, T. (2013). Revealed preference theory for finite choice sets. Discussion papers ces13.08, Katholieke Universiteit Leuven, Centrum voor Economische Studin.

Diewert, W. E. (1973). Afriat and revealed preference theory. Review of Economic Studies, 40(3), 419-25.

Echenique, F., Lee, S., and Shum, M. (2011a). The money pump as a measure of revealed preference violations. Journal of Political Economy, 119(6), 1201 - 1223.

Echenique, F., Golovin, D., and Wierman, A. (2011b). A revealed preference approach to computational complexity in economics. In Proceedings of the 12th ACM conference on Electronic commerce, EC '11, pages 101-110, New York, NY, USA. ACM.

Ekeland, I. and Galichon, A. (2012). The housing problem and revealed preference theory: duality and an application. Economic Theory. Forthcoming.

Forges, F. and Iehlé, V. (2012). Essential data, budget sets and rationalization. Economic Theory. Forthcoming.

Forges, F. and Minelli, E. (2009). Afriat's theorem for general budget sets. Journal of Economic Theory, 144(1), 135-145.

Fostel, A., Scarf, H., and Todd, M. (2004). Two new proofs of Afriat's theorem. Economic Theory, 24(1), 211-219. 
Fujishige, S. and Yang, Z. (2012). On revealed preference and indivisibilities. Modern Economy, 3(6), $752-758$.

Mas-Colell, A., Whinston, M., and Green, J. (1995). Microeconomic Theory. Oxford University Press.

Polisson, M. and Quah, J. (2013). Revealed preference in a discrete consumption space. American Economic Journal: Microeconomics, 5(1), 28-34.

Sákovics, J. (2013). Revealed cardinal preference. Economic Theory Bulletin, 1, 39-45.

Varian, H. R. (1982). The nonparametric approach to demand analysis. Econometrica, 50(4), $945-73$.

Varian, H. R. (1990). Goodness-of-fit in optimizing models. Journal of Econometrics, 46(1-2), $125-140$. 